\title{
THE WHITHAM EQUATION FOR HYDROELASTIC WAVES
}

\author{
EVGUENI DINVAY, HENRIK KALISCH, DAULET MOLDABAYEV, AND EMILIAN I. PĂRĂU
}

\begin{abstract}
A weakly nonlinear fully dispersive model equation is derived which describes the propagation of waves in a thin elastic body overlying an incompressible inviscid fluid. The equation is nonlocal in the linear part, and is similar to the so-called Whitham equation which was proposed as a model for the description of wave motion at the free surface of an inviscid fluid.

Steady solutions of the fully nonlinear hydro-elastic Euler equations are approximated numerically, and compared to numerical approximations to steady solutions of the fully dispersive but weakly nonlinear model equation.

The bifurcation curves for these two different models are compared, and it is found that the weakly nonlinear model gives accurate predictions for waves of small to moderate amplitude. For larger amplitude waves, the two models still agree on key qualitative features such as the bifurcation points, secondary bifurcations, and the number of oscillations in a given fundamental wave period.
\end{abstract}

\section{INTRODUCTION}

The present contribution is devoted to hydroelastic waves propagating along a thin elastic body overlying an incompressible inviscid fluid. The prime example for this situation is wave propagation in an ice sheet over a body of water, a topic which has attracted increasing attention among researchers in recent years.

One of the motivating problems for studying this situation has been the motion of trucks and other vehicles on frozen lakes and rivers (see for example [27, 35]). In many such cases, hydroelastic waves are highly dispersive but only weakly nonlinear. For example the measurements recorded in [35] feature a large spectrum of wavelengths while nonlinearity is relatively weak. As a result, many researchers have chosen to disregard nonlinear effects altogether [10, 25, 27, 34]. More recently, nonlinear effects have come into focus as some studies of weakly and fully nonlinear hydroelastic waves (see for example [30, 29, 40]) have suggested that nonlinearity does have an appreciable effect on hydro-elastic waves.

The nonlinear model system derived in [40] couples the well known Saint-Venant (shallow water) system with hydroelastic dispersion, but neglects gravity dispersion. Going further, the works $[14,15]$ take into account gravity dispersion in addition to both elasticity and nonlinearity. These studies depend heavily on the long-wave assumption and therefore lose part of the information of the linearized problem, as wavelengths are restricted to be very long when compared with the undisturbed depth of the fluid and the elastic length scale. In order to improve the modeling accuracy of such long-wave systems, in the present work we are aiming at the derivation of a fully dispersive but weakly nonlinear system. As mentioned above, the motivation and need for such a system is given by experiments such as those reported in [35] where a weakly nonlinear but highly dispersive response is recorded which may not be adequately modeled by traditional long-wave equations.

Date: March 25, 2019. 
The idea of using fully dispersive weakly nonlinear equations goes back to Whitham [38], and was recently formalized both mathematically [21, 22, 23] and asymptotically $[11,26]$. Fully dispersive equations have been the subject of a number of studies recently, especially regarding the existence and stability of traveling waves $[4,16,17,18,19,33]$. In the current work, we first present a formal derivation of a Whitham-type fully dispersive and weakly nonlinear system of evolution equations, and then reduce the system to a single equation in the case when it can be assumed that the waves travel in a single direction (such as is the case for traveling waves). Using a recently developed open-source Python code, we then provide numerical approximations of traveling-wave solutions of this system. In order to check whether the derivation and numerical approximation are valid, we compare these solutions against numerical approximations of traveling-waves solutions of the fully nonlinear Euler equations with an elastic surface layer. As will come to light in Section 4, there is very good agreement between solutions of the full model and the weakly nonlinear model.

The fully dispersive weakly nonlinear equation we are aiming for can be written as

$$
\eta_{t}+W \eta_{x}+\frac{3}{2} \eta \eta_{x}=0
$$

Elasticity and gravity dispersion are combined in the convolution Whitham operator $W \eta_{x}=w\left(-i \partial_{x}\right) \eta_{x}=\left(\mathcal{F}^{-1} w\right) * \eta_{x}$ which is defined by the dispersive function

$$
w(\xi)=\sqrt{\left(1+\varkappa \xi^{4}\right) \frac{\tanh (\xi)}{\xi}} .
$$

Here we have non-dimensionalized the variables so that we may take the gravitational acceleration $g=1$ and the undisturbed depth of the fluid as $h_{0}=1$, and the corresponding long-wave speed is $c_{0}=\sqrt{g h_{0}}=1$. This non-dimensionalization is explained in the next section. The floating ice sheet is included here by means of the non-zero elasticity parameter $\varkappa=\mathcal{D} / \rho$ where $\mathcal{D}$ is the coefficient of flexural rigidity for the ice sheet and $\rho=1$ is the normalized density of the fluid. The convolution operator $W$ represents a Fourier multiplier operator with the symbol (1.2). Note also that the Whitham equation (1.1) has the conserved integral

$$
Q(\eta)=\int \eta W \eta d x+\frac{1}{2} \int \eta^{3} d x .
$$

Firstly, in case of free surface $\varkappa=0$, Equation (1.1) was proposed by Whitham [38] as a fully dispersive alternative to the well known Korteweg-de Vries (KdV) equation. With non-zero $\varkappa$ the last equation [14] is of the fifth order

$$
\eta_{t}+\eta_{x}+\frac{3}{2} \eta \eta_{x}+\frac{1}{6} \eta_{x x x}+\frac{1}{360}(19+180 \varkappa) \eta_{x x x x x}=0 .
$$

For very long waves the KdV equation (1.4) is thought to be a good model for hydroelastic waves. In order to be able to model shorter waves one needs to use an equation which also gives a good description of shorter waves, such as for example equation (1.1).

In the present article, we arrive at the Whitham equation from the Hamiltonian formulation of the water-wave problem given in $[41,9,7]$ and modified for the hydroelastic problem in [14]. Extending the results of $[26,14,11]$ we justify the Whitham equation (1.1) as a Hamiltonian system. We also obtain numerical results on steady solutions of equation (1.1) and compare them with solutions of the fully nonlinear hydro-elastic system based on the Euler equations. The comparisons indicate that the Whitham equation is able to provide a good description of hydro-elastic waves. Indeed, small-amplitude 
solutions of the full Euler equations are always closely approximated by the weakly nonlinear model, regardless of the wavelength. For large-amplitude solutions, there is good quantitative agreement in many cases, and good qualitative agreement in essentially all cases.

\section{THE HYDRO-ELASTIC SYSTEM}

We consider a thin elastic plate supported by a fluid below. The elastic layer is modelled by making use of the special Cosserat theory of hyperelastic shells in Cartesian coordinates [31]. As already stated, the fluid base is assumed to be inviscid and incompressible, and the fluid flow is assumed to be two-dimensional and irrotational, so that potential theory can be used to describe the flow.

In order to normalize the problem the following standard non-dimensionalization is used. Letting the dimensional variables be primed, the non-dimensional variables are defined in terms of the fluid depth $h_{0}$, the graviational acceleration $g$ and the long-wave speed $c_{0}=\sqrt{g h_{0}}$ as $x^{\prime}=h_{0} x, z^{\prime}=h_{0} x$ and $t^{\prime}=\sqrt{h_{0} / g} t$. The unknowns are the vertical deflection of the cover and the velocity potential, and these are nondimensionalized as $\eta^{\prime}=h_{0} \eta$ and $\phi^{\prime}=h_{0} c_{0} \phi$, respectvely. This non-dimensionalization is equivalent to using the fluid depth $h_{0}$ as a unit of length, and $\sqrt{h_{0} / g}$ as a unit of time.

In the non-dimensional setting, the fluid domain is given by $\left\{(x, z) \in \mathbb{R}^{2} \mid-1<z<\eta(x, t)\right\}$ extending to infinity in the positive and negative horizontal $x$-direction. The complete hydro-elastic Euler system [14] consists of the Laplace's equation in this domain

$$
\phi_{x x}+\phi_{z z}=0 \quad \text { for } \quad x \in \mathbb{R}, \quad-1<z<\eta(x, t),
$$

the Neumann boundary condition at the flat bottom

$$
\phi_{z}=0 \text { at } z=-1
$$

the kinematic condition at the free surface

$$
\eta_{t}+\phi_{x} \eta_{x}-\phi_{z}=0 \quad \text { for } \quad x \in \mathbb{R}, \quad z=\eta(x, t),
$$

and the Bernoulli equation combined with elasticity

$$
\phi_{t}+\frac{1}{2}\left(\phi_{x}^{2}+\phi_{z}^{2}\right)+\eta+\varkappa\left(\kappa_{s s}+\frac{1}{2} \kappa^{3}\right)=0 \quad \text { for } \quad x \in \mathbb{R}, \quad z=\eta(x, t),
$$

where $\kappa=\eta_{x x}\left(1+\eta_{x}^{2}\right)^{-3 / 2}$ is the curvature of the shell and $s$ is the arclength along this cover and therefore

$$
\kappa_{s s}+\frac{1}{2} \kappa^{3}=\frac{1}{\sqrt{1+\eta_{x}^{2}}} \partial_{x}\left(\frac{1}{\sqrt{1+\eta_{x}^{2}}} \partial_{x}\left(\frac{\eta_{x x}}{\left(1+\eta_{x}^{2}\right)^{3 / 2}}\right)\right)+\frac{1}{2}\left(\frac{\eta_{x x}}{\left(1+\eta_{x}^{2}\right)^{3 / 2}}\right)^{3} .
$$

The total energy of the system is the sum of the kinetic energy, the potential energy and the shell deformation energy [14], so the Hamiltonian function for this problem is expressed as

$$
H=\int_{\mathbb{R}} \int_{0}^{\eta} z d z d x+\int_{\mathbb{R}} \int_{-1}^{\eta} \frac{1}{2}|\nabla \phi|^{2} d z d x+\frac{\varkappa}{2} \int_{\mathbb{R}} \frac{\eta_{x x}^{2}}{\left(1+\eta_{x}^{2}\right)^{5 / 2}} d x .
$$

Introducing the trace of the potential at the free surface as $\Phi(x, t)=\phi(x, \eta(x, t), t)$, one may integrate in $z$ in the first integral and use the divergence theorem on the second integral to obtain

$$
H=\frac{1}{2} \int_{\mathbb{R}}\left[\eta^{2}+\Phi G(\eta) \Phi+\varkappa \frac{\eta_{x x}^{2}}{\left(1+\eta_{x}^{2}\right)^{5 / 2}}\right] d x
$$


This integral represents the Hamiltonian functional of the water wave problem with a floating thin elastic cover as found in [14]. The Hamiltonian is written in terms of the Dirichlet-Neumann operator $G(\eta)$. It was shown in [28] that this operator depends analytically on the unknown $\eta$ and can therefore be expanded in a power series

$$
G(\eta) \Phi=\sum_{j=0}^{\infty} G_{j}(\eta) \Phi,
$$

where each operator $G_{j}(\eta)$ is homogeneous of degree $j$ in $\eta$. The terms in the power series can be computed using a recursion formula (see $[9,7]$ ), and the first two terms have the form

$$
G_{0}(\eta)=D \tanh (D), \quad G_{1}(\eta)=D \eta D-D \tanh (D) \eta D \tanh (D)
$$

where $D=-i \partial_{x}$ is a self-adjoint operator on $L^{2}(\mathbb{R})$.

Our first aim is to give the Hamiltonian formulation of the hydroelastic problem in terms of the displacement $\eta$ and the variable $u=\Phi_{x}=\phi_{x}+\eta_{x} \phi_{y}=\phi_{\tau} \sqrt{1+\eta_{x}^{2}}$ proportional to the velocity of the fluid $\phi_{\tau}$ tangential to the surface. Formally integrating by parts one may rewrite the Hamiltonian in terms of new variables as

$$
H=\frac{1}{2} \int_{\mathbb{R}}\left[\eta^{2}+u D^{-1} G(\eta) D^{-1} u+\varkappa \frac{\eta_{x x}^{2}}{\left(1+\eta_{x}^{2}\right)^{5 / 2}}\right] d x .
$$

The integration by parts can be made mathematically precise by using the following well known lemma.

Lemma 2.1. Let $f, g$ be real-valued square integrable functions on the real line $\mathbb{R}$. Regard $D=-i \partial_{x}$ as self-adjoint on $L^{2}(\mathbb{R}, \mathbb{C})$ and a real-valued function $\varphi$ that is measurable and almost everywhere finite with respect to Lebesgue measure. If $f, g$ lie in the domain of the operator $\varphi(D)$ then

$$
\int f \varphi(D) g=\int g \varphi(-D) f
$$

\section{Derivation of Whitham type evolution equations}

We now give a formal analysis of the long-wave approximation of the Hamiltonian. For the sake of completeness, we summarize some arguments presented in [11]. Regarding a wave-field having a characteristic non-dimensional wavelength $\lambda$ and a characteristic non-dimensional amplitude $\alpha$, we define the small parameter $\mu=\frac{1}{\lambda}$ and in order to bring out the difference in the horizontal and vertical scales in the problem, the scalings $\eta=\alpha \tilde{\eta}$ and $\tilde{D}=\lambda D=-\lambda i \partial x$ are used. Then the natural scaling for the velocity unknown $u$ is $u=\alpha \tilde{u}$, and the Hamiltonian is scaled as $H=\alpha^{2} \tilde{H}$. Omitting terms of cubic and higher order in $\alpha$, the scaled Hamiltonian (2.6) is then written using only the terms $G_{1}$ and $G_{2}$ as

$$
\begin{aligned}
& \tilde{H}=\frac{1}{2} \int_{\mathbb{R}} \tilde{\eta}^{2} d x+\frac{1}{2} \int_{\mathbb{R}} \tilde{u}\left[1-\frac{1}{3} \mu^{2} \tilde{D}^{2}+\cdots\right] \tilde{u} d x+\frac{\alpha}{2} \int_{\mathbb{R}} \tilde{\eta} \tilde{u}^{2} d x \\
&-\frac{\alpha}{2} \int_{\mathbb{R}} \tilde{u}\left[\mu \tilde{D}-\frac{1}{3} \mu^{3} \tilde{D}^{3}+\cdots\right] \tilde{\eta}\left[\mu \tilde{D}-\frac{1}{3} \mu^{3} \tilde{D}^{3}+\cdots\right] \tilde{u} d x \\
&+\mu^{4} \frac{\varkappa}{2} \int_{\mathbb{R}}\left(\tilde{D}^{2} \tilde{\eta}\right)^{2}\left\{1-\alpha^{2} \mu^{2} \frac{5}{2}(\tilde{D} \tilde{\eta})^{2}+\cdots\right\} d x .
\end{aligned}
$$

In the case of flexural-gravity waves, the linear terms are dominant and terms of all order in $\mu$ should be kept. We may assume that $\alpha=o(\mu)$ such as for example the exponential 
scaling appearing in [26]. Then if terms of order $O\left(\alpha^{2}\right)$ and $O(\alpha \mu)$ are disregarded, the Hamiltonian (2.6) reduces to

$$
H=\frac{1}{2} \int_{\mathbb{R}}\left[\eta^{2}+u \frac{\tanh D}{D} u+\eta u^{2}+\varkappa \eta_{x x}^{2}\right] d x .
$$

Now the hydro-elastic problem can be reformulated as a Hamiltonian system using the variational derivatives of the approximate Hamiltonian $(3.1)$. We point out $[7,8]$ that the pair $(\eta, \Phi)$ represents the canonical variables for the Hamiltonian functional (2.5). Our purpose is to derive the equations of motion in terms of $\eta$ and $u=\Phi_{x}$ which is slightly more convenient in the situation at hand. The transformation $(\eta, \Phi) \mapsto(\eta, u)$ is associated with the Jacobian

$$
\frac{\partial(\eta, u)}{\partial(\eta, \Phi)}=\left(\begin{array}{cc}
1 & 0 \\
0 & \partial_{x}
\end{array}\right)
$$

So in terms of $\eta$ and $u$ the Hamiltonian equations have the form

$$
\eta_{t}=-\partial_{x} \frac{\delta H}{\delta u}, \quad u_{t}=-\partial_{x} \frac{\delta H}{\delta \eta}
$$

which is not canonical since the associated structure map $J_{\eta, u}$ is symmetric:

$$
J_{\eta, u}=\left(\frac{\partial(\eta, u)}{\partial(\eta, \Phi)}\right)\left(\begin{array}{cc}
0 & 1 \\
-1 & 0
\end{array}\right)\left(\frac{\partial(\eta, u)}{\partial(\eta, \Phi)}\right)^{*}=\left(\begin{array}{cc}
0 & -\partial_{x} \\
-\partial_{x} & 0
\end{array}\right) .
$$

The Gâteaux derivative $\delta H / \delta u$ of the Hamiltonian (3.1) is defined by means of an arbitrary real-valued square integrable function $h$ from the variation

$$
\delta_{u} H(h)=\left.\frac{d}{d \tau}\right|_{\tau=0} H(u+\tau h, \eta)=\frac{1}{2} \int_{\mathbb{R}}\left[h \frac{\tanh D}{D} u+u \frac{\tanh D}{D} h+2 u \eta h\right] d x .
$$

Integrating by parts according to Lemma 2.1 and taking into account arbitrariness of the given function $h$ one obtains

and similarly

$$
\frac{\delta H}{\delta u}=\frac{\tanh D}{D} u+\eta u
$$

$$
\frac{\delta H}{\delta \eta}=\eta+\frac{1}{2} u^{2}+\varkappa \eta_{x x x x} .
$$

Substituting these variational derivatives into equations (3.2) we arrive at the Hamiltonian system

$$
\begin{aligned}
& \eta_{t}=-\frac{\tanh D}{D} u_{x}-(\eta u)_{x}, \\
& u_{t}=-\eta_{x}-u u_{x}-\varkappa \eta_{x x x x x} .
\end{aligned}
$$

This system is fully dispersive in the linear part, which is the distinctive feature of the original Whitham equation [38]. At the same time it allows bi-directional wave propagation, so that it may be of independent interest.

By changing variables in the Whitham system (3.3),(3.4) one may arrive at a new system, where the equations will be uncoupled in the linear part. In other words we can separate solutions corresponding to waves moving left and right. To justify this we follow the approach of $[11,26]$, and consider the linearization

$$
\begin{aligned}
\eta_{t}+\frac{\tanh D}{D} u_{x} & =0, \\
u_{t}+\left(1+\varkappa D^{4}\right) \eta_{x} & =0 .
\end{aligned}
$$


Looking for solutions of this linear system as waves $\eta(x, t)=A e^{i \xi x-i \omega t}, u(x, t)=B e^{i \xi x-i \omega t}$ gives rise to the necessary condition $\omega^{2}-\left(\xi+\varkappa \xi^{5}\right) \tanh \xi=0$. Introducing the phase speed as $c=\omega(\xi) / \xi$ one arrives at the dispersion relation

$$
c^{2}(\xi)=\left(1+\varkappa \xi^{4}\right) \frac{\tanh \xi}{\xi}
$$

which coincides, up to the sign of $c$, with the Whitham dispersion relation (1.2). Clearly, the choice $c>0$ corresponds to right-going wave solutions of the linear system (3.5), (3.6), while the phase speed $c<0$ gives left-going waves. In order to split up left- and right-going waves we use the following transformation of variables:

$$
r=\frac{1}{2}(\eta+K u), \quad s=\frac{1}{2}(\eta-K u)
$$

where one anticipates $K$ to be an invertible operator, or more precisely, an invertible function of the differential operator $D$. The inverse transformation has the form

$$
\eta=r+s, \quad u=K^{-1}(r-s) .
$$

It turns out that the operator $K$ can be chosen in a way that $r$ and $s$ correspond to rightand left-going waves, respectively. Substituting the transformation (3.8) to the linear system (3.5), (3.6) obtain

$$
\begin{aligned}
& r_{t}+\partial_{x}(\mathcal{A}(D, K) r+\mathcal{B}(D, K) s)=0, \\
& s_{t}-\partial_{x}(\mathcal{A}(D, K) s+\mathcal{B}(D, K) r)=0,
\end{aligned}
$$

where the operators $\mathcal{A}$ and $\mathcal{B}$ depend on $D$ and $K$ as follows:

$$
\mathcal{A}=\frac{1}{2}\left(\left(1+\varkappa D^{4}\right) K+\frac{\tanh D}{D} K^{-1}\right), \quad \mathcal{B}=\frac{1}{2}\left(\left(1+\varkappa D^{4}\right) K-\frac{\tanh D}{D} K^{-1}\right) .
$$

So to achieve independence of the obtained two equations we need to choose the transformation $K$ in the way $\mathcal{B}(D, K)=0$, so that

$$
K=\sqrt{\frac{1}{1+\varkappa D^{4}} \cdot \frac{\tanh D}{D}}
$$

which leads to $\mathcal{A}(D, K)=W=w(D)$, in terms of the dispersive Whitham operator $W$ which was introduced at the beginning of the paper by means of the dispersive function (1.2). In this way, we get the two independent linear equations

$$
\begin{aligned}
& r_{t}+\partial_{x} W r=0, \\
& s_{t}-\partial_{x} W s=0 .
\end{aligned}
$$

If we again look at the special solutions $r(x, t)=\exp \left(i \xi x-i \omega_{r} t\right)$ and $s(x, t)=\exp \left(i \xi x-i \omega_{s} t\right)$ then we conclude that the first equation (3.10) describes waves moving to the right with the phase velocity $c_{r}=\omega_{r} / \xi=w(\xi)$ and the second equation (3.11) corresponds to the left-going waves with $c_{s}=\omega_{s} / \xi=-w(\xi)$.

Returning to the nonlinear theory we want to obtain a new Hamiltonian system with respect to unknown functions (3.7). In new variables $r$ and $s$ after integrating by parts due to Lemma 2.1 the Hamiltonian (3.1) takes the form

$$
\begin{aligned}
H(r, s)=\frac{1}{2} \int_{\mathbb{R}}\left[(r+s)^{2}+(r-s)(1+\right. & \left.\varkappa D^{4}\right)(r-s)+ \\
& \left.+(r+s)\left(K^{-1}(r-s)\right)^{2}+\varkappa(r+s)_{x x}^{2}\right] d x .
\end{aligned}
$$


As explained in [8], the change of variables (3.7) transforms the structure map to

$$
J_{r, s}=\left(\frac{\partial(r, s)}{\partial(\eta, u)}\right) J_{\eta, u}\left(\frac{\partial(r, s)}{\partial(\eta, u)}\right)^{*}=\left(\begin{array}{cc}
-\frac{1}{2} \partial_{x} K & 0 \\
0 & \frac{1}{2} \partial_{x} K
\end{array}\right)
$$

that leads to the Hamiltonian system

$$
r_{t}+\partial_{x}\left(\frac{K}{2} \frac{\delta H}{\delta r}\right)=0, \quad s_{t}-\partial_{x}\left(\frac{K}{2} \frac{\delta H}{\delta s}\right)=0 .
$$

As explained above, one calculates variational derivatives of $H$ given by (3.12) with respect to $r$ and $s$ at a real-valued square integrable function. Then after integrating by parts as in Lemma 2.1 and applying operator $K / 2$ one obtains as a result

$$
\begin{aligned}
& \frac{K}{2} \frac{\delta H}{\delta r}=W r+\frac{1}{4} K\left(K^{-1}(r-s)\right)^{2}+\frac{1}{2}(r+s) K^{-1}(r-s), \\
& \frac{K}{2} \frac{\delta H}{\delta s}=W s+\frac{1}{4} K\left(K^{-1}(r-s)\right)^{2}-\frac{1}{2}(r+s) K^{-1}(r-s),
\end{aligned}
$$

If these expressions are substituted into (3.13), a Whitham system describing in terms of a left-going component $s$ and a right-going compnent $r$ is appears. This system corresponds to the Hamiltonian (3.12) which is the same as for the system (3.3), (3.4), and no further approximation has been made. Recalling that the approximate Hamiltonian (3.12) was obtained by discarding terms of order $O(\mu \alpha)$ and $O\left(\alpha^{2}\right)$ in (2.6) we may modify terms in the Hamiltonian on the of order $O(\mu \alpha)$ and $O\left(\alpha^{2}\right)$ without changing the overall order of approximation.

Thus in order to simplify the above system, we use the long-wave approximation $D=$ $O(\mu)$ which leads to $K=1+O\left(\mu^{2}\right), K^{-1}=1+O\left(\mu^{2}\right)$ and since $r=O(\alpha), s=O(\alpha)$, the nonlinear part of (3.14), (3.15) can be written as

$$
H_{1}=\frac{1}{2} \int_{\mathbb{R}}(r+s)\left(K^{-1}(r-s)\right)^{2} d x=\frac{1}{2} \int_{\mathbb{R}}(r+s)(r-s)^{2} d x+O\left(\mu^{2} \alpha\right) .
$$

Thus neglecting again terms of order $O(\mu \alpha)$, and assuming that the left-going waves are $s=o(\alpha)$ such as in [7], we can write the nonlinear part of the Hamiltonian as $H_{1}=1 / 2 \int_{\mathbb{R}} r^{3} d x$. So eventually we arrive at

$$
\begin{gathered}
\frac{K}{2} \frac{\delta H}{\delta r}=W r+\frac{3}{4} K r^{2}, \\
\frac{K}{2} \frac{\delta H}{\delta s}=W s .
\end{gathered}
$$

These approximations in the Hamiltonian together with the equations (3.13) yield the system

$$
\begin{aligned}
r_{t}+W r_{x}+\frac{3}{2} K\left(r r_{x}\right) & =0, \\
s_{t}-W s_{x} & =0 .
\end{aligned}
$$

This system is a fully dispersive system describing waves mainly moving in the right direction. Since we have modified only the energy of the hydro-elastic problem, the system (3.18), (3.19) is still Hamiltonian. Indeed that system corresponds to the Hamiltonian

$$
H(r, s)=\frac{1}{2} \int_{\mathbb{R}}\left[(r+s)^{2}+(r-s)\left(1+\varkappa D^{4}\right)(r-s)+r^{3}+\varkappa(r+s)_{x x}^{2}\right] d x .
$$

As one can see the first equation (3.18) can be considered independently from the second one (3.19) which is actually linear and homogeneous. Moreover, one can easily see that 
the Hamiltonian splits additively into two functionals $H(r, s)=H(r)+H(s)$, where the first one has the form

$$
H(r)=\int_{\mathbb{R}}\left[r\left(1+\varkappa D^{4}\right) r+\frac{1}{2} r^{3}\right] d x .
$$

With this energy, comparable with the initial total energy (2.5), the new Whitham equation (3.18) becomes Hamiltonian in the sense (3.13).

One may now use the approximation $K=1+O\left(\mu^{2}\right)$ in the first equation (3.18) to arrive at the Whitham equation (1.1). This is consistent with disregarding terms of order $O(\alpha \mu)$ in the derivation of the system (3.3),(3.4). Note, however, that this equation is then not Hamiltonian in the current context. Indeed, we have changed the structure map $J_{r, s}$, and the unknown $\eta$ in equation (1.1) has the same meaning as $r$ in equation (3.18), though the Hamiltonian structure is different. We have

$$
\eta_{t}+\partial_{x}\left(\frac{1}{2} \frac{\delta Q}{\delta \eta}\right)=0
$$

where $Q(\eta)$ is given in (1.3). Note that energy $Q$ is not comparable with the original Hamiltonian $H$ given in (2.5), and it is therefore not clear whether it represents the energy. Indeed, as indicated in [1], the mechanical energy might take a completely different form in the context of equation (1.3).

\section{NUMERICAL ANALYSIS}

For the numerical analysis of equation (1.1) we use the method thoroughly described in $[20,32]$. Here we will just give a quick overview. Travelling wave solutions are under investigation, so the ansatz

$$
\eta(x, t)=\varphi(x-c t)
$$

is employed which together with equation (1.1) yields

$$
-c \varphi+\frac{3}{4} \varphi^{2}+W \varphi=B
$$

with some undetermined constant $B$. We look for solutions of the last equation that are even and $2 L$-periodic, so we compute solutions on the interval $[0, L]$ and restore it symmetrically on the whole interval $[-L, L]$. We regard solutions with different wavelengths $L$ and elasticity $\varkappa$. We also normalize solutions by requiring its mean-value to be zero. This means that we require $\int_{0}^{L} \varphi=0$. We use a cosine collocation method, so solutions of equation (4.2) are represented as linear combinations of functions $\cos \frac{m \pi x}{L}, m=0,1, \ldots$, that form a basis in $L^{2}(0, L)$. As explained in [17, 20], for the discretization of the problem we look for solutions in a subspace $\mathcal{S}_{N}$ spanned by the first $N$ of these cosine functions and defined at the collocation points $x_{n}=L \frac{2 n-1}{2 N}$ for $n=1, \ldots, N$. Let $W^{N}$ be the discrete form of the Whitham operator $W$ and $\varphi_{N} \in \mathcal{S}_{N}$ be the discrete cosine representation of a solution $\varphi$. Then the values of $\varphi_{N}$ at the collocation points satisfy the algebraic equations

$$
-c \varphi_{N}\left(x_{n}\right)+\frac{3}{4} \varphi_{N}^{2}\left(x_{n}\right)+W^{N} \varphi_{N}\left(x_{n}\right)=B
$$

with $n=1, \ldots, N$. As indicated above, to this system one also needs to add the meanvalue normalization

$$
\sum_{n=1}^{N} \varphi_{N}\left(x_{n}\right)=0 .
$$


To compute profiles along the bifurcation curve, we introduce a parameter $\theta$ as a coordinate on the curve. We define the pseudo-waveheight $A$ by the equation

$$
\max _{n=1, \ldots, N} \varphi_{N}\left(x_{n}\right)-\min _{n=1, \ldots, N} \varphi_{N}\left(x_{n}\right)=A,
$$

and treat the pseudo-waveheight $A=A(\theta)$ and the phase speed $c=c(\theta)$ as functions of the parameter $\theta$. This procedure is described in more detail in [20].

Equations (4.3), (4.4), (4.5) form the final system to be solved by Newton's method. The unknowns here are $\varphi_{N}\left(x_{1}\right), \ldots, \varphi_{N}\left(x_{N}\right), B, \theta$. Solutions bifurcating from the linearization of equation (4.2) can be obtained from the initial guess, provided by the Stokes approximation [20].

The numerical algorithm used to solve the fully nonlinear Euler equations with an elastic term (2.1) - (2.4) is based on the conformal mapping techniques [13, 12] and generalizes previous works on gravity waves or capillary waves $[6,2]$ to the hydroelastic case [14]. We will give just some essentials here. The Bernoulli equation (2.4) at the free surface in the moving frame $X=x-$ ct and $Y=z$ has the form

$$
\frac{1}{2}\left(\phi_{X}^{2}+\phi_{Y}^{2}\right)+\eta+\varkappa\left(\kappa_{s s}+\frac{1}{2} \kappa^{3}\right)=\mathcal{B}
$$

where $\mathcal{B}$ is the Bernoulli constant. As above (4.1) the surface is represented by $\eta(x, t)=$ $\varphi(X)$, so the outward unit normal vector is $\mathbf{n}=\left(-\varphi_{X}, 1\right) / \sqrt{1+\varphi_{X}^{2}}$.

The physical domain bounded by the free surface and the bottom is transformed with a conformal mapping from $X+i Y$ to $\xi+i \zeta$ into a strip of thickness $h$ (called conformal modulus), with $\zeta=0$ corresponding to the free surface and $\zeta=-h$ corresponding to the bottom. Solving a Dirichlet boundary-value problem for $Y(\xi, \zeta)$ in the mapped domain, using Cauchy-Riemann relations between the partial derivatives of $X(\xi, \zeta)$ and $Y(\xi, \zeta)$ and denoting $\hat{X}(\xi):=X(\xi+i 0) \mathrm{m} \hat{Y}(\xi):=Y(\xi+i 0)$, we obtain after some algebra and dropping the hats (see e.g. [6])

$$
X_{\xi}=1-\mathcal{T} Y_{\xi}
$$

where $\mathcal{T}$ is the operator $\mathcal{T}=i \operatorname{coth} h D$. In the new variables the Bernoulli equation can be re-written

$$
\frac{1}{2} \frac{c^{2}}{X_{\xi}^{2}+Y_{\xi}^{2}}+Y+\varkappa\left(\kappa_{s s}+\frac{1}{2} \kappa^{3}\right)=\mathcal{B} .
$$

The elastic term $\left(\kappa_{s s}+\frac{1}{2} \kappa^{3}\right)$ can be expanded in an explicit form in terms of derivatives of $X$ and $Y$, as explained in [15]. The velocity potential on the free surface is satisfying $\phi=c \xi$. We expand the variable $Y$ as a Fourier series

$$
Y(\xi)=\sum_{n=-\infty}^{\infty} a_{n} e^{i n \xi},
$$

and we are using equally-spaced collocation points $\xi_{j}=\frac{2 \pi(j-1)}{2 N+1}, j=1, \ldots, 2 N+1$. The non-local operator acts (for $n \neq 0$ ) as

$$
\mathcal{T}\left(e^{i n \xi}\right)=i \operatorname{coth}(n h) e^{i n \xi} .
$$

After truncating the Fourier series at $n= \pm N$, we satisfy the equation (4.6) at the $2 N+1$ collocation points for the $2 N+2$ unknowns $a_{n}, n=-N, \ldots, N$ and the Bernoulli constant $\mathcal{B}$. The remaining equation is obtained by fixing $a_{0}=h$ and the nonlinear system obtained is solved in MATLAB using a Newton method. In practice, for any given $c$ we choose an initial guess $h=1$ of the conformal modulus and then we enforce that the 


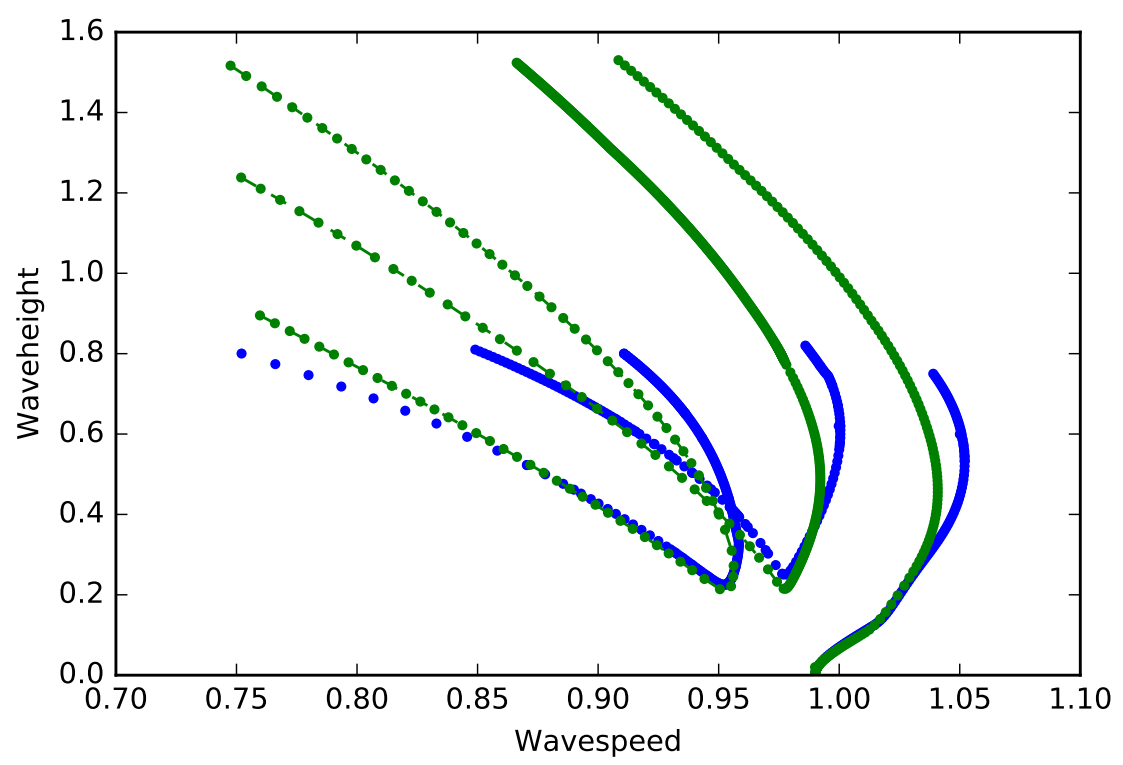

FiguRE 1. Bifurcation diagrams for $L=4 \pi$ and $\varkappa=0.1$. The blue curves represent the full hydroelastic system. The green curves represent the Whitham equation.

dimensionless height of the fluid in the physical space remain unchanged and equal to 1 for all computations, so we actually use a fixed-point procedure to guarantee

$$
\frac{1}{2 L} \int_{-L}^{L} Y X_{\xi} d \xi=1,
$$

until the correct value of $h$ is found for each $c$. It was found that $N=128$ is enough for most of the calculations to obtain a very good accuracy of solutions. When the curves are turning in the bifurcation diagrams, a version of this algorithm is used, where the height of the wave is enforced and $c$ was found as part of the solution.

In figures 1 through 18, one can see how solutions change in the bifurcation diagrams. Solutions are plotted on the interval $[-L, L]$, where $L$ ranges from $\pi / 2$ to $4 \pi$, and several features are worth noting. First of all, in the bifurcation curves in figures 1, 8 and 11, for large to intermediate wavelengths, the bifurcation curves of the hydro-elastic Whitham equation are very close to the bifurcation curves for the Euler system as long as the waveheight, defined as $\max (Y)-\min (Y)$, is below about 0.4. A similar conclusion is reached by examining figures 2, 9, 12 and 16, where steady wave profiles are shown, and a close match between the two curves is evident. Whitham waves of larger amplitude still resemble the full hydro-elastic waves, qualitatively as shown in figures 3, 4, 5, 6, 7 for $L=4 \pi$ and $\varkappa=1$, and similarly in figures 13,14 and 18 for other parameter values. It is also worth noting that on the period $[-4 \pi, 4 \pi]$ solutions with large enough waveheight develop several secondary crests which actually start dominating the main crest at the center of the wave.

For small-amplitude solutions the error in the phase speed $c$ is generally below $1 \%$. For large-amplitude solutions, the error in the phase speed becomes larger. An extreme example is shown in figures 8 and 10. Concerning solutions high up on the branch in Figure 8, if one matches the waveheight, then a near-exact match in the shape of the wave is obtained, such as in the blue and green profiles in Figure 10. However, the phase 


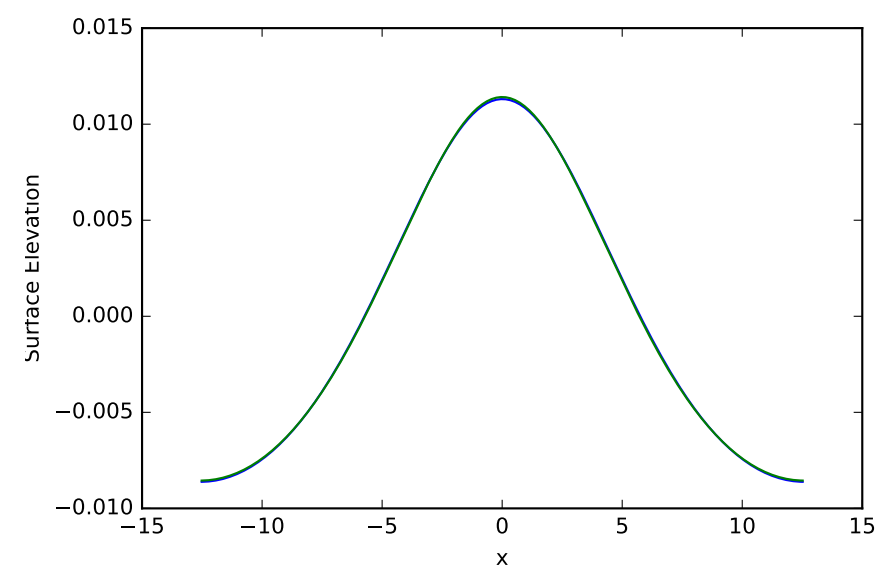

FiguRE 2. Wave profiles for $L=4 \pi, \varkappa=0.1$ near the bifurcation point of the rightmost curve in Figure 1. The blue curve is an approximate solution of the full Euler system with $c=0.9910$. The green curve an approximate solution of the Whitham equation with $c=0.9911$.

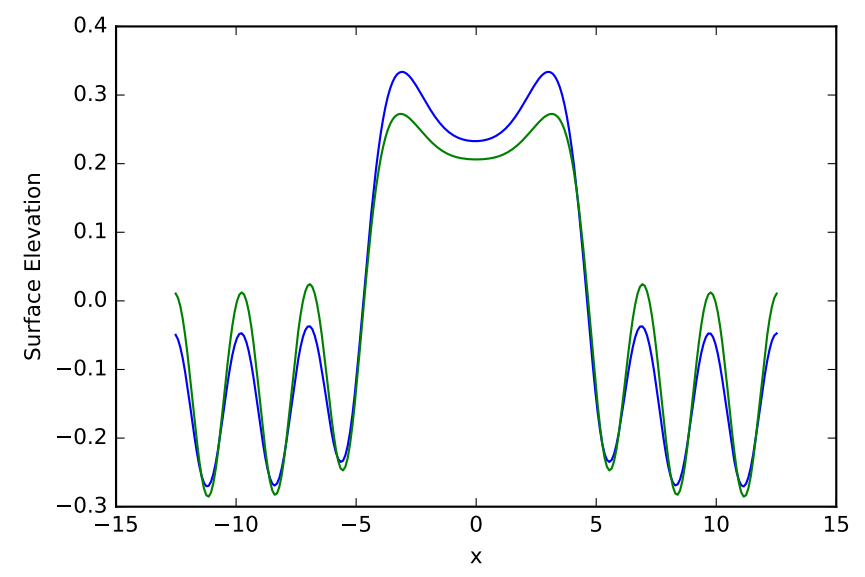

FiguRE 3. Wave profiles for $L=4 \pi, \varkappa=0.1$ higher up on the main branch in Figure 1. The blue curve represents the Euler system with $c=1.0506$. The green curve represents the Whitham equation with $c=1.0391$.

velocities for these two solutions vary a great deal. On the other hand if one tries to get the best fit for the phase velocity, then the solution profiles have very different waveheights, though they still look qualitatively similar (cf. blue and red curve in Figure 10). Figure 10 also shows the interesting result (when compared with figures 13 and 14) that a larger elastic parameter $\varkappa$ actually leads to fewer oscillations in the steady wave profile.

For large enough wavelengths and small enough elasticity, branches were found which do not connect to trivial solutions with vanishing waveheight (see figures 1 and 11). On the other hand, for short wavelengths there are secondary bifurcation from the main branch, such as shown in Figure 15. In this figure, one can see how $2 \pi$-periodic solutions bifurcate from $\pi$-periodic ones. In all cases examined, the bifurcation picture of the Whitham model looks qualitatively similar to the fully nonlinear hydro-elastic system. Since the Whitham equation is much more easily approximated numerically, future work 


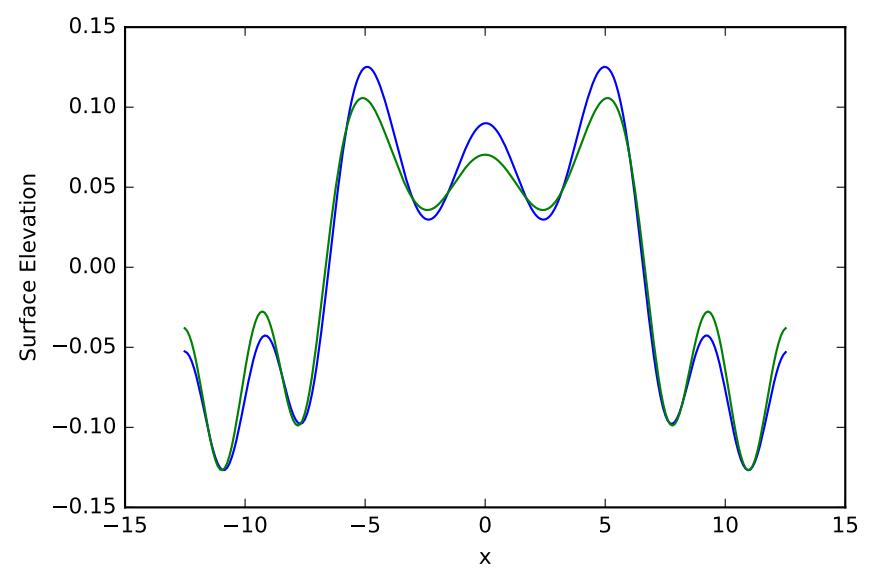

FIgURE 4 . Wave profiles for $L=4 \pi, \varkappa=0.1$ on the second (middle) bifurcation point in Figure 1. The blue curve represents the Euler system with $c=0.9764$. The green curve represents the Whitham equation with $c=0.9742$.

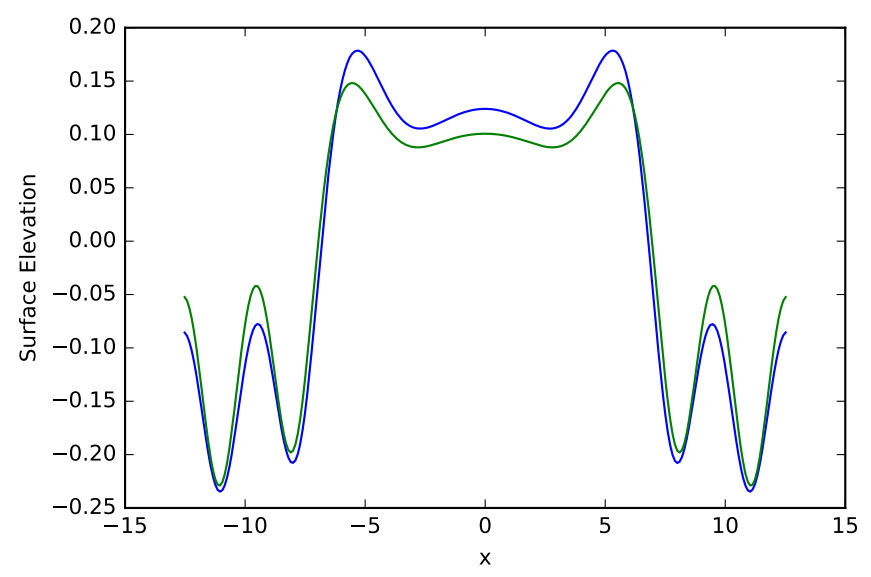

FiguRE 5. Wave profiles for $L=4 \pi, \varkappa=0.1$ on the left bifurcation branch emanating from the middle bifurcation point in Figure 1. The blue curve represents the Euler system with $c=0.9553$. The green curve represents the Whitham equation with $c=0.9545$.

will include obtaining bifurcation curves for a larger parameter space of $\varkappa$ and $L$, such as for example presented for the similar Whitham equation with capillarity in [32].

Finally, we want to comment on two other models mentioned in the current paper, the 5th-order KdV equation (1.4) with a flexural term, and the fully nonlinear Whitham equation (3.18). Since the flexural effect corresponds to the term with the 5 th derivative in (1.4), it appears that elasticity has a rather weak effect in the long-wave regime. Indeed, in the case of long waves, the Whitham equation (1.1) with a free surface (i.e. $\varkappa=0$ ) gives similar small-amplitude solutions to solutions of other models depicted in Figure 12. However, with increasing of amplitude elasticity causes the appearance of several crests on the fundamental wavelength $[-L, L]$, whereas the wave profiles of the free surface Whitham equation have exactly one crest and are strictly monotonic to both sides of it [17]. Some simulations of (1.4) and (3.18) are presented in Figures 11-14. Note that both Whitham equations (1.1), (3.18) give travelling waves very similar to the fully nonlinear 


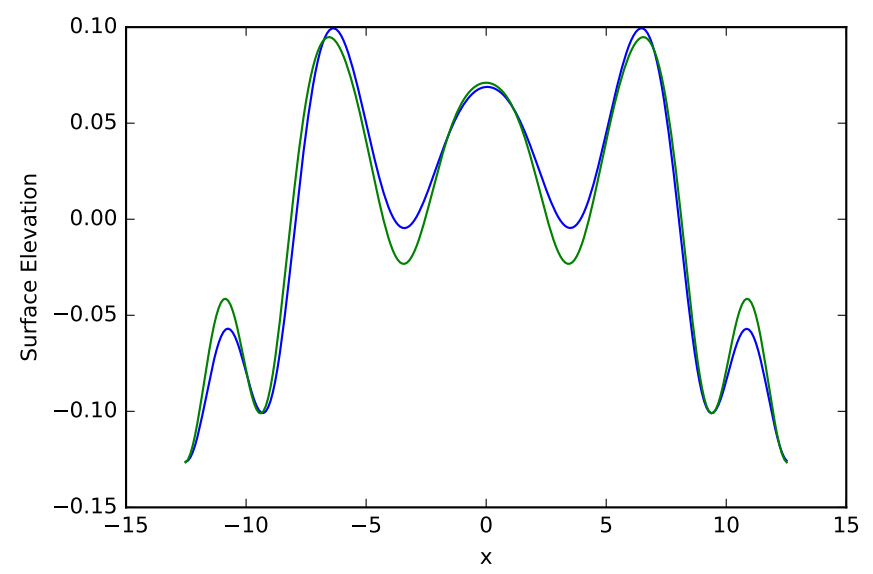

FiguRE 6 . Wave profiles for $L=4 \pi, \varkappa=0.1$ on the third (leftmost) bifurcation point shown in Figure 1. The blue curve represents the Euler system with $c=$ 0.9530. The green curve represents the Whitham equation with $c=0.9552$.

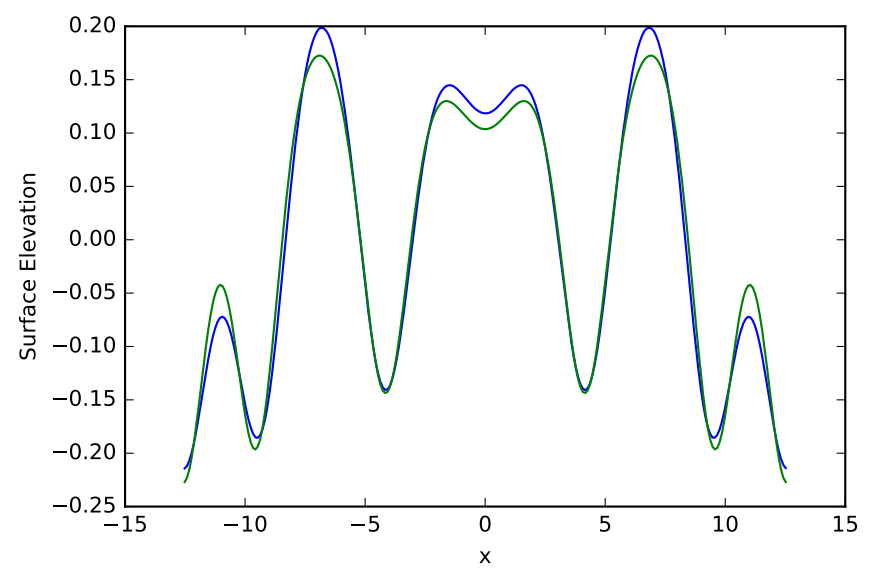

FigURE 7 . Wave profiles for $L=4 \pi, \varkappa=0.1$ on the right bifurcation branch emanating from the leftmost bifurcation point in Figure 1. The blue curve represents the Euler system with $c=0.9553$. The green curve represents the Whitham equation with $c=0.9501$.

solutions. Whereas the elastic KdV (1.4) gives qualitatively different waves, i. e. with a different number of of wavecrests on a fundamental wavelength. Moreover, the KdV wave depicted in Figure 14 is a solution of the secondary bifurcation type. It can be obtained by bifurcating from the $L=\pi$-branch as shown in Figure 11. On the other hand, it does not seem possible to obtain corresponding solutions of the full system and equations (1.1), (3.18) in a similar way continuously from linear theory.

\section{Conclusion}

In order to understand properties of waves in a thin elastic sheet overlaying an inviscid fluid, a fully dispersive system of equations was derived using the Hamiltonian formulation of the hydroelastic surface wave system. The Hamiltonian was approximated using an asymptotic expansion of the Dirichlet-Neumann operator, and the derivation was based on fundamental ideas regarding Hamiltonian evolution systems [7, 8, 9, 41]. It was also shown 


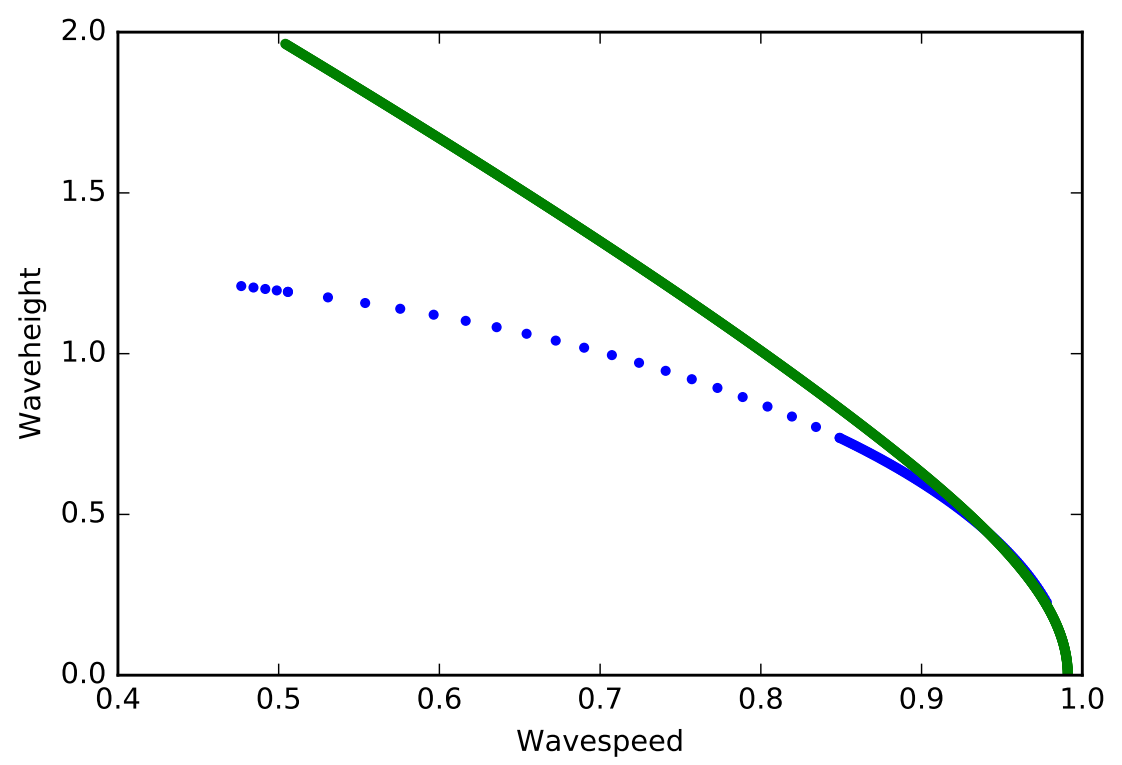

FiguRE 8. Bifurcation diagrams for $L=2 \pi$ and $\varkappa=1$. The blue curve represents the full hydroelastic system. The green curve represents the Whitham equation.

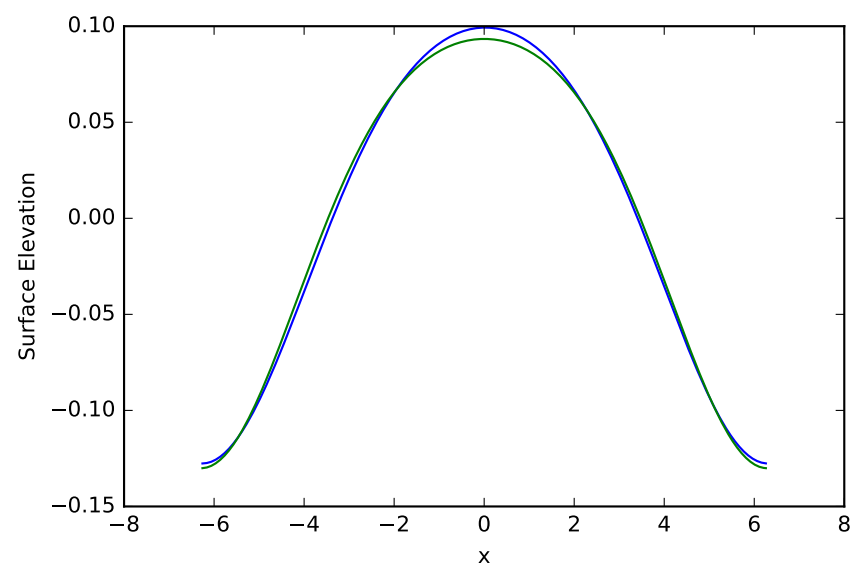

FigURE 9. Wave profiles for $L=2 \pi, \varkappa=1$ for small-amplitude solutions on the bifurcation branches in Figure 8. The blue curve represents the Euler system with $c=0.9779$. The green curve represents the Whitham equation with $c=$ 0.9771 .

how the Hamiltonian equations can be restricted to model wave propagation in a single direction. Finally, a single fully dispersive weakly nonlinear equation of Whitham type (1.1) has been derived. This equation is the hydro-elastic version of the so-called Whitham equation put forward by Whitham [38] based on phenomenological considerations.

In order to further validate equation (1.1) as a model for hydro-elastic waves, a numerical study of traveling waves has been undertaken. Numerical approximations of steady solutions of (1.1) were compared to numerical approximations of steady solutions of the full hydro-elastic system introduced in Section 2. Bifurcation branches and wave profiles 


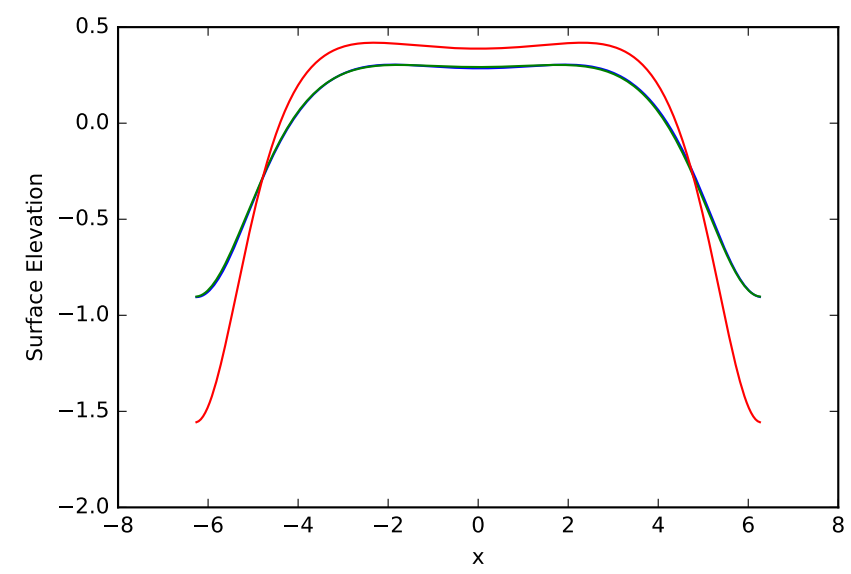

FiguRE 10. Wave profiles for $L=2 \pi, \varkappa=1$. The blue curve represents the Euler system with $c=0.4767$. The green curve represents the Whitham equation with $c=0.7431$. The red curve shows a different solutions of the Whitham equation with $c=0.5002$ (that is $5 \%$ difference from $c=0.4767$ ).

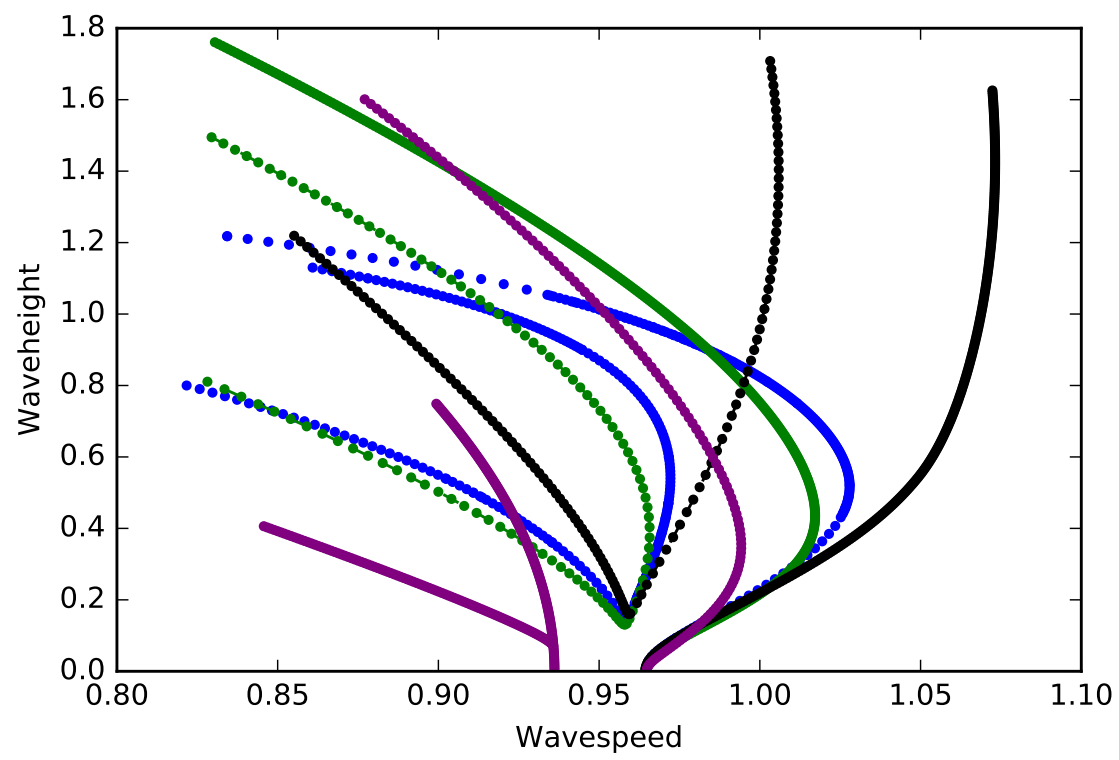

FiguRE 11. Bifurcation diagrams for $L=2 \pi$ and $\varkappa=0.1$. The blue curves represent the hydroelastic system. The green curves represent the Whitham equation (1.1). The black curves correspond to the fully nonlinear Whitham equation (3.18). The purple color is associated with the KdV-like equation (1.4).

were compared, and it was shown that small-amplitude solutions of (1.1) resemble solutions of the full hydro-elastic system rather closely. Intermediate-amplitude solutions of the two approximations are still comparable, and even large-amplitude solutions of (1.1) show good qualitative agreement. In addition, it can be seen in figures 1, 8, 11 and 15 that bifurcation branches of (1.1) contain the same features as the bifurcation curves for the corresponding fully nonlinear hydro-elastic system. In particular, primary and secondary bifurcation points and the number of branches are closely matched. 


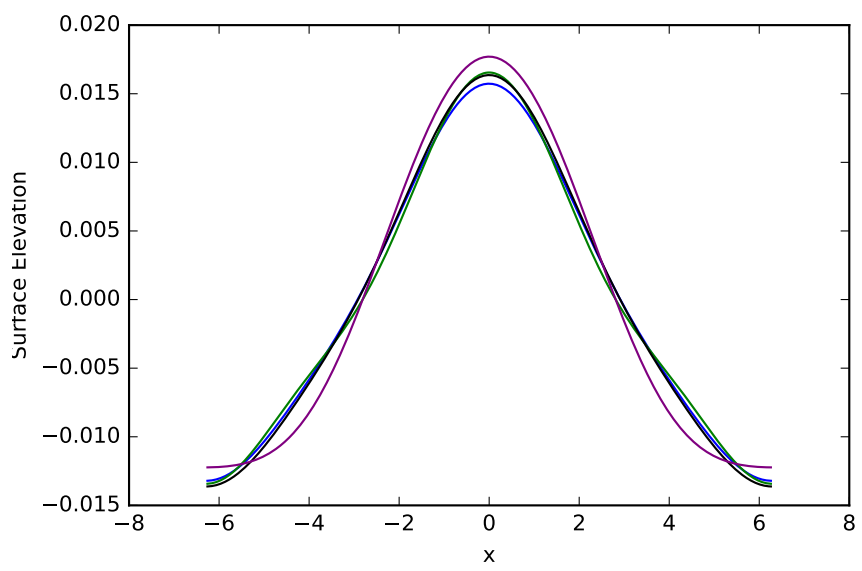

FiguRE 12. Wave profiles for $L=2 \pi, \varkappa=0.1$ for small-amplitude waves on the main branch in Figure 11. The blue curve shows a solution of the full hydro-elastic system with $c=0.9653$. The green curve shows a solution of the Whitham equation (1.1) with $c=0.9657$. The black solution corresponds to the Hamiltonian Whitham equation (3.18) with $c=0.9654$. The purple color is associated with the KdV equation (1.4) for $c=0.9668$.

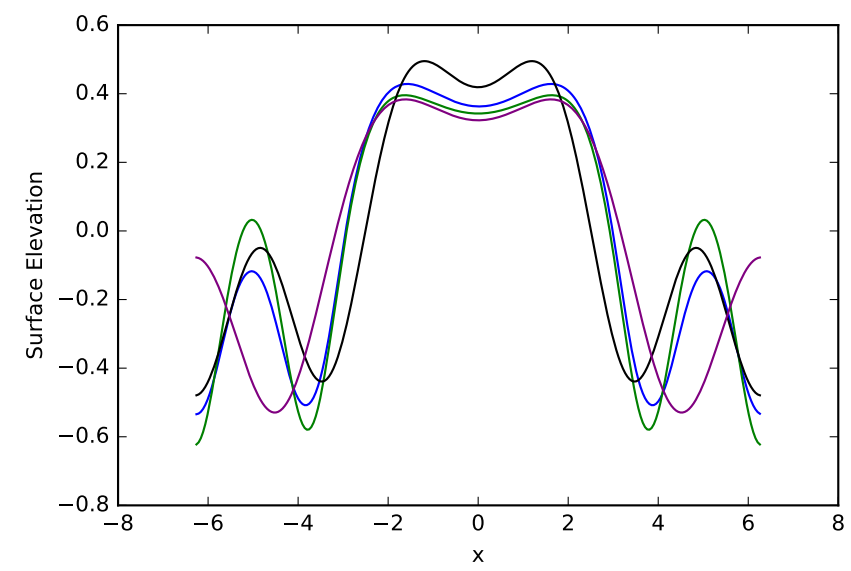

FIgURE 13. Wave profiles for $L=2 \pi, \varkappa=0.1$ for waves high up on the main branch in Figure 11. The blue curve shows a solution of the full hydro-elastic system with $c=0.9668$. The green curve shows a solution of the Whitham (1.1) equation with $c=0.9685$. The black solution corresponds to the Hamiltonian Whitham equation (3.18) with $c=1.0682$. The purple color is associated with the $\mathrm{KdV}$ equation (1.4) for $c=0.9607$.

Further work on these issues will include the study of whether the flow below the surface can be reconstructed from the surface profile $\eta(x, t)$. Such an analysis has recently been begun in the case of a free surface in [3]. In the present situation, this issue is more complicated because of the elastic layer. Nevertheless, analyzing for example the derivation used in [40] it seems possible that progress could be made.

Another important issue which deserves further study is the analysis of the nonlinear regime of the bifurcation curves, and the stability of the traveling waves. Such an analysis 


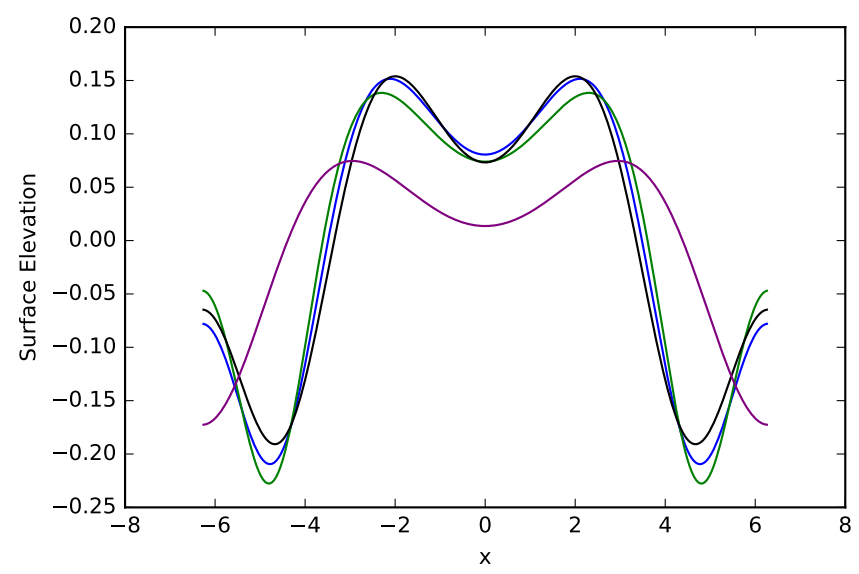

FiguRE 14. Wave profiles for $L=2 \pi, \varkappa=0.1$ on the leftmost branch in Figure 11. The blue curve shows a solution of the full hydro-elastic system with $c=0.9353$. The green curve shows a solution of the Whitham equation (1.1) with $c=0.9261$. The black solution corresponds to the Hamiltonian Whitham equation (3.18) with $c=0.9486$. The purple color is associated with the $\mathrm{KdV}$ equation (1.4) for $c=0.8932$. It corresponds to the secondary bifurcation curve in Figure 11.

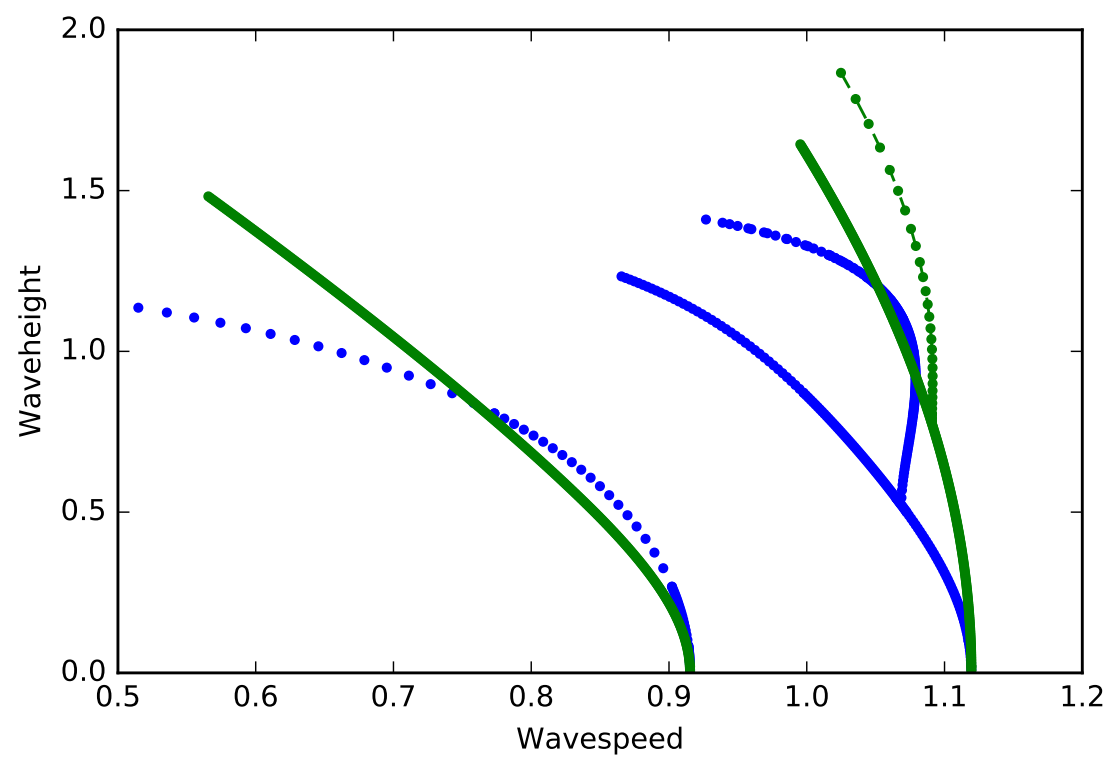

FiguRE 15. Bifurcation diagrams for $L=\pi$ and $L=\pi / 2$ with $\varkappa=0.1$. The blue curves represent the full hydroelastic system. The green curves represent the Whitham equation.

has been provided for the original Whitham equation [4, 18, 33], and the Whitham equation with surface tension in $[19,32]$ but not yet for the equation derived in the current work.

The current model may also be extended by the inclusion of a variety of additional physical effects, such as a horizontal loading on the ice sheet, as described in [40], a 


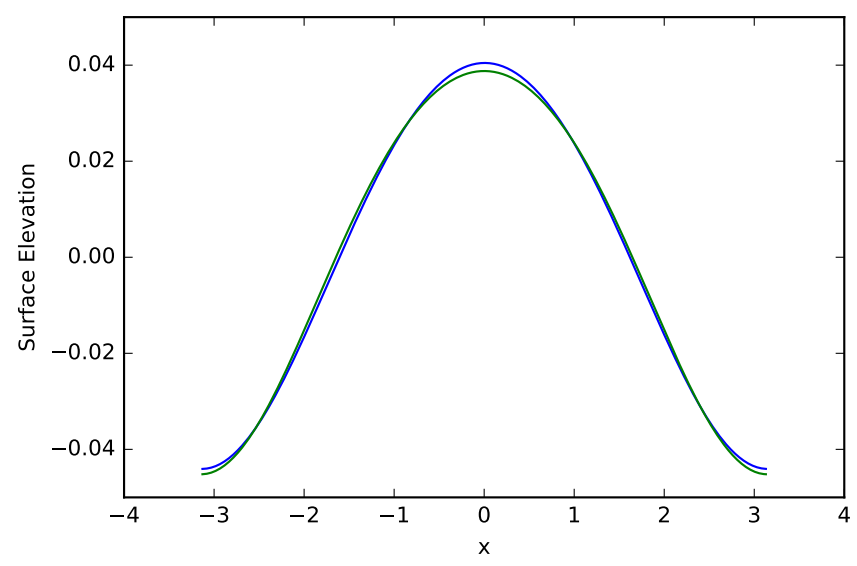

FiguRE 16. Wave profiles for $L=\pi, \varkappa=0.1$ for small-amplitudes solutions on the left branch in Figure 15. The blue curve shows a solution of the full hydro-elastic system with $c=0.9140$. The green curve shows a solution of the Whitham equation with $c=0.9129$.

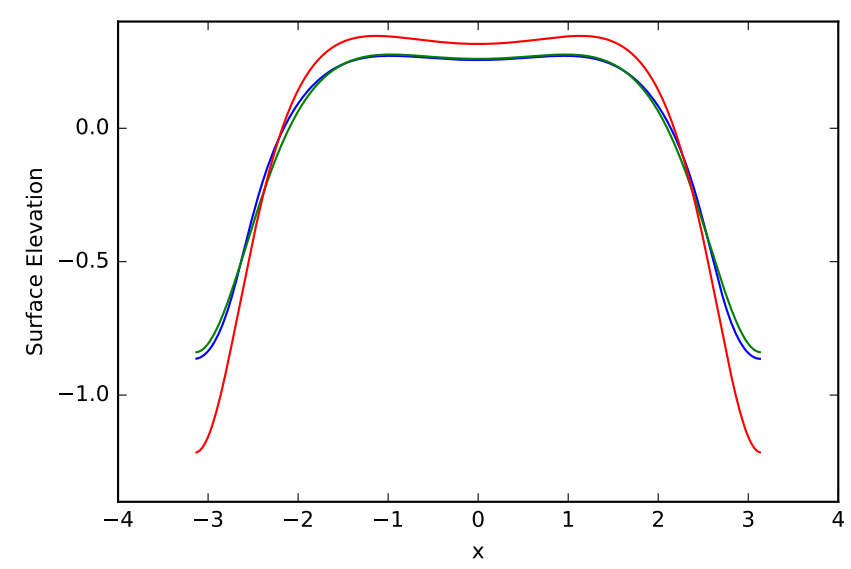

FiguRE 17. Wave profiles for $L=\pi, \varkappa=0.1$ on the left branch in Figure 15 . The blue curve shows a solution of the full hydro-elastic system with $c=0.5148$. The green curve shows a solution of the Whitham equation with $c=0.6790$. The red curve shows a solution of the Whitham equation with $c=0.5402$ (that is $5 \%$ difference from $c=0.5148$ ).

vertical forcing [35], and the influence of nontrivial bathymetry [37]. These issues will be the focus of future work.

Acknowledgments. This research was supported by the Research Council of Norway under grant no. 213747/F20 and 239033/F20. E.I. Părău has been partially supported by the EPSRC under grant EP/J019305/1.

\section{REFERENCES}

[1] Ali, A. and Kalisch, H. On the formulation of mass, momentum and energy conservation in the KdV equation, Acta Appl. Math. 133 (2014), 113-131.

[2] Blyth, M. and Părău, E.I. The stability of capillary waves on fluid sheets. J. Fluid Mech. 806 (2016), $5-34$. 


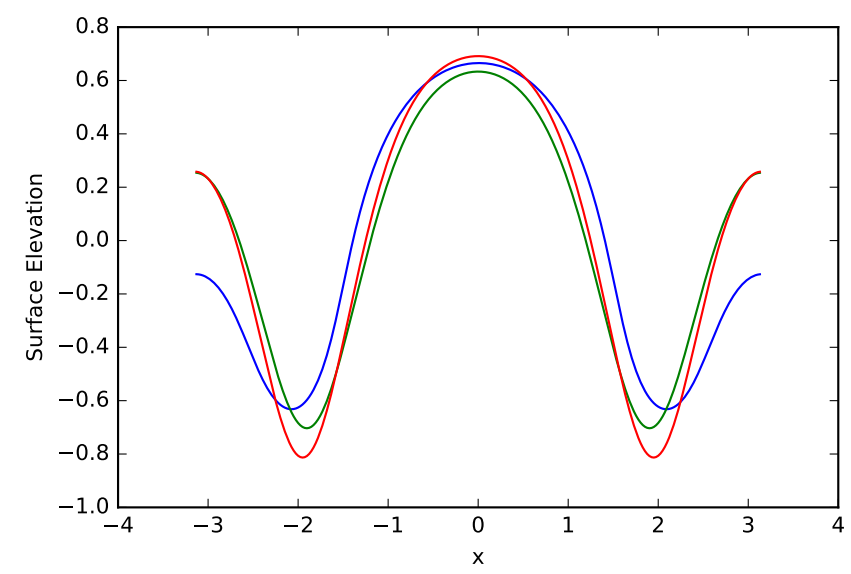

FiguRE 18. Wave profiles for $L=\pi, \varkappa=0.1$ on the secondary branch on the very right in Figure 15. Note that these solutions have full wavelength $2 \pi$, the same as the solutions in the leftmost branch in Figure 15. The blue curve shows a solution of the full hydro-elastic system with $c=1.0173$. The green curve shows a solution of the Whitham equation with $c=1.0786$. The red curve shows a solution of the Whitham equation with $c=1.0658$ (that is $5 \%$ difference from $c=1.0173)$.

[3] H. Borluk and H. Kalisch, Particle dynamics in the KdV approximation, Wave Motion 49 (2012), 691-709.

[4] Bronski, J.C., Hur, V.M. and Johnson, M.A. Modulational instability in equations of KdV type, New Approaches to Nonlinear Waves. Springer International Publishing, 2016. 83-133.

[5] Carter, J.D. Bidirectional Whitham equations as models of waves on shallow water, arXiv:1705.06503 (2017).

[6] Choi, W. and Camassa, R. Exact Evolution Equations for Surface Waves. J. Eng. Mech. 125 (1999), $756-760$.

[7] Craig, W. and Groves, M.D. Hamiltonian long-wave approximations to the water-wave problem. Wave Motion 19 (1994), 367-389.

[8] Craig, W., Guyenne, P. and Kalisch, H. Hamiltonian long-wave expansions for free surfaces and interfaces. Comm. Pure Appl. Math. 58 (2005), 1587-1641.

[9] Craig, W. and Sulem, C. Numerical simulation of gravity waves. J. Comp. Phys. 108 (1993), 73-83.

[10] Davys, J.W. Hosking, R.J. and Sneyd, A.D. Waves due to a steadily moving source on a floating ice plate, J. Fluid Mech. 158 (1985), 269-287.

[11] Dinvay, E., Moldabayev, D., Dutykh, D. and Kalisch, H. The Whitham equation with surface tension, Nonlinear Dynamics 88 (2017), 1125-1138.

[12] Dyachenko, A.I., Zakharov, V.E., and Kuznetsov, E.A. Nonlinear dynamics of the free surface of an ideal fluid, Plasma Phys. Rep., 22 (1996), 829-840.

[13] Dyachenko, A.I., Kuznetsov, E.A., Spector, M.D., and Zakharov, V.E. Analytical description of the free surface dynamics of an ideal fluid (canonical formalism and conformal mapping), Phys. Lett., A, 221 (1996), 73-79.

[14] Guyenne, P. and Părău E.I. Finite-Depth Effects on Solitary Waves in a Floating Ice-Sheet, J. Fluids and Structures 49, 242-262 (2014).

[15] Guyenne, P. and Părău, E.I. Computations of fully-nonlinear hydroelastic solitary waves on deep water, J. Fluid Mech. 713 (2012), 307-329.

[16] Ehrnström, M., Kalisch, H. Traveling waves for the Whitham equation. Diff. Int. Eq. 22 (2009), $1193-1210$

[17] Ehrnström, M., Kalisch, H. Global bifurcation for the Whitham equation. Math. Modelling Natural Phenomena 8 (2013), 13-30.

[18] Hur, V.M. and Johnson, M. Modulational instability in the Whitham equation of water waves. Studies in Applied Mathematics 134 (2015), 120-143. 
[19] Hur, V.M. and Johnson, M. Modulational instability in the Whitham equation with surface tension and vorticity. Nonlinear Anal. 129 (2015), 104-118.

[20] Kalisch, H., Moldabayev D. and Verdier O. A numerical study of nonlinear dispersive wave models with SpecTraVVave, Electronic Journal of Differential Equations. 2017: 1-23.

[21] Klein, C., Linares, F., Pilod, D. and Saut, J.-C. On Whitham and related equations, Stud. Appl. Math. 140 (2018), 133-177.

[22] Lannes, D. The Water Waves Problem. Mathematical Surveys and Monographs, vol. 188 (Amer. Math. Soc., Providence, 2013).

[23] Lannes, D. and Saut, J.-C. Remarks on the full dispersion Kadomtsev-Petviashvli equation. Kinet. Relat. Models 6 (2013), 989-1009.

[24] Linares, F., Pilod, D. and Saut, J.-C. Dispersive perturbations of Burgers and hyperbolic equations I: local theory. SIAM J. Math. Anal. 46 (2014), 1505-1537.

[25] Milinazzo, F., Shinbrot, M. and Evans, N.W. A mathematical analysis of the steady response of floating ice to the uniform motion of a rectangular load, J. Fluid Mech. 287 (1995), 173-197.

[26] Moldabayev, D., Kalisch, H. and Dutykh, D. The Whitham Equation as a model for surface water waves, Phys. D 309 (2015), 99-107.

[27] Nevel, D.E. Moving loads on a floating ice sheet, CRREL Res. Rep. 261. (1970), US Army Cold Regions Research and Engineering Laboratory, Hanover, NH, USA.

[28] Nicholls, D.P. and Reitich, F. A new approach to analyticity of Dirichlet-Neumann operators. Proc. Roy. Soc. Edinburgh Sect. A 131 (2001), 1411-1433.

[29] Părău E.I. and Vanden-Broeck, J.-M. Three-dimensional waves beneath an ice sheet due to a steadily moving pressure, Phil. Trans. Roy. Soc. A: 369 (2011), 2973-2988.

[30] Părău E. and Dias, F. Nonlinear effects in the response of a floating ice plate to a moving load, J. Fluid Mech. 460 (2002), 281-305.

[31] Plotnikov, P.I. and Toland, J.F. Modelling nonlinear hydroelastic waves, Phil. Trans. R. Soc. A 369 (2011) 2942-2956;

[32] Remonato, F. and Kalisch, H. Numerical bifurcation for the capillary Whitham equation, Physica D 343 (2017), 51-62.

[33] Sanford, N., Kodama, K., Carter, J.D. and Kalisch, H. Stability of traveling wave solutions to the Whitham equation. Phys. Lett. A 378 (2014), 2100-2107.

[34] Schulkes, R.M.S.M. and Sneyd, A.D. Time-dependent response of floating ice to a steadily moving load, J. Fluid Mech. 186 (1988), 25-46.

[35] Takizawa, T. Field studies on response of a floating sea ice sheet to a steadily moving load, Contrib. Inst. Low Temp. Sci. A 36 (1987), 31-76.

[36] Takizawa, T. Response of a floating sea ice sheet to a steadily moving load, J. Geophys. Res. 93 (1988), 5100-5112.

[37] Vargas-Magana, R.M. and Panayotaros, P. A Whitham-Boussinesq long-wave model for variable topography, Wave Motion 65 (2016), 156-174.

[38] Whitham, G.B. Variational methods and applications to water waves. Proc. Roy. Soc. London A 299 (1967), 6-25.

[39] Whitham, G.B. Linear and Nonlinear Waves (Wiley, New York, 1974).

[40] Xia, X. and Shen, H.T. Nonlinear interaction of ice cover with shallow water waves in channels, J. Fluid Mech. 467 (2002), 259-268.

[41] Zakharov, V.E. Stability of periodic waves of finite amplitude on the surface of a deep fluid. J. Appl. Mech. Tech. Phys. 9 (1968), 190-194.

EVGueni.dinVay@math.uib.no, henrik.kalisch@math.uib.no, Department of Mathematics, University of Bergen, Postbox 7800, 5020 Bergen, Norway.

daulet.moldabayev@math.uib.no, Oceaneering Asset Integrity AS, PB 1228, Sluppen, 7462 Trondheim, NORWAY.

e.parau@uea.ac.uk, School of Mathematics, University of East Anglia, Norwich ReSEARCH PARK, Norwich, NR4 7TJ, United Kingdom 